\title{
Astrocyte Dysfunctions and HIV-1 Neurotoxicity
}

Hoai Ton and Huangui Xiong*

Neurophysiology Laboratory, Department of Pharmacology and Experimental Neuroscience, University of Nebraska Medical Center, Omaha, NE 68198-5880, USA

\begin{abstract}
Astrocytes play an important role in maintaining an optically suited milieu for neuronal functionality, and are involved in the progression and outcome of many neuropathological conditions. It becomes increasingly evident that astrocytes are significant contributors to HIV-1 associated neurological disorders by modulating the microenvironment in the central nervous system and releasing proinflammatory cytokines. Recent studies have revealed direct metabolic interactions between neurons and astrocytes observed particularly in HIV-1-associated neurological disorders by which astrocytic dysfunctions disregulate extracellular $\mathrm{K}+$ homeostasis, intracellular calcium concentration, glutamate clearance, and blood brain barrier integrity and permeability. Such dysfunctions are amplified via gap junctions, directly or indirectly impacting surrounding neurons and significantly contributing to the pathogenesis of HIV-1-associated neuropathology. In this review, we tentatively address recent progresses on the roles astrocytes may play in HIV-1associated neurotoxicity.
\end{abstract}

Keywords: Astrocytes; HIV-1 associated neurotoxicity; K+ channels; Glutamate uptake; Blood brain barrier; Gap junctions

\section{Introduction}

The human immunodeficiency virus type one (HIV-1) invades the central nervous system (CNS) at early stage after primary infection [1], which may result ultimately in the development of several types of neurological disorders. Such HIV-1-associated neurological disorders affect about $50 \%$ of infected individuals [2]. Characteristics of neurological disorders encompass cognitive disturbances, behavioral changes, and motor impairment [3], which are termed collectively as HIV-1-associated neurocognitive disorders (HAND) [4]. Despite the advent of combined antiretroviral therapy (cART), the prevalence of HAND continues to rise due to sustained HIV-1-infection of mononuclear phagocytes (MP, brain macrophages and microglia), the emergence of resistant viral phenotypes, poor drug penetration of blood-brain barrier and increase in patient lifespan. This indicates that HAND remains highly prevalent and continues to be a significant public health problem [5-7]. As cART cannot provide complete protection from HIV-1-induced neuronal injury and there is no efficacious treatment for HAND at present, mainly because the precise mechanisms underlying HIV-induced neuronal injury are not well understood. Thus, investigating molecular and cellular mechanisms of HIV-1-induced neuronal injury may provide valuable insights into the pathogenesis of HAND, and is essential for the development of preventive and therapeutic strategies.

The cellular basis and mechanisms underlying HAND is complex and have so far remained elusive. It has long been believed that neuropathogenesis of HAND revolves solely around metabolic processes induced by virus-infected and activated MPs. This is underscored by the findings that the abundance of macrophages in the brain appears to better correlate with HAND than the extent of brain infection [8,9], implicating that an increase in trafficking of macrophages to the brain may be associated with the development of HAND. In addition to pathogenic role of MPs, it is becoming increasing evident that astrocytes are also important players in the pathogenesis of HAND and other neurological disorders.

Astrocytes are the most abundant cell type within the CNS and play an important role in CNS homeostasis and function. They are also the target cells for immune mediators, bacterial toxins and viruses (such as HIV-1) that induce reactive astrogliosis, a common feature seen in many neurological disorders including HAND [10-12]. Astrocytes respond to pathological challenges by rapid activation, not only at the site of challenge but also in the surrounding neuropil [13]. Studies have shown an up-regulated expression of many proteins in activated astrocytes. These include cytokines, growth factors, cell surface molecules and extracellular matrix proteins [11]. While many of these molecules function as neurotrophic agents under certain circumstance, ample evidence support an important role for astrocytes as mediators of HIV-1-induced neuronal injury $[11,14,15]$. However, the mechanisms for how activated astrocytes induced neuronal injury are not fully understood. In this article, we try to highlight astrocytic dysfunctions and their roles in HIV-1 neurotoxicity, which potentially contribute to the pathogenesis of HIV-1 associated neurocognitive impairment.

\section{Astrocytosis}

Astrocytosis, activated astrocytes, is an abnormal increase in the number and size of astrocytes in response to the CNS injury and disease. It has been observed in brain trauma, infection, stroke and other neurodegenerative diseases. Astrocytosis is characterized by hypertrophy, cell proliferation, progressive alteration in molecular expression and up-regulation of glial fibrillary acidic protein (GFAP) expression [16]. Astrocytosis may be beneficial or harmful in proximity to neural and non-neural cells. They undergo a spectrum of changes that may alter astrocytic activities and affecting surrounding cells.

HIV-1 infection of astrocytes has been noted in pediatric, and to a lesser extent, adult HIV-1 associated encephalopathy. Such infection is restricted at both virus entry and post-entry viral gene expression [17]. It has been shown that in HIV-1 infected individuals astrocytosis can be triggered not only by virus infection [18], but also induced by

*Corresponding author: Huangui Xiong, Department of Pharmacology and Experimental Neuroscience, University of Nebraska Medical Center, Omaha, NE 681985880, USA, Tel: 402-559-5140; Fax: 402-559-3744; E-mail: hxiong@unmc.edu

Received July 29, 2013; Accepted November 12, 2013; Published November 19, 2013

Citation: Ton H, Xiong H (2013) Astrocyte Dysfunctions and HIV-1 Neurotoxicity. J AIDS Clin Res 4: 255. doi: 10.4172/2155-6113.1000255

Copyright: ( $)$ 2013Ton $\mathrm{H}$, et al. This is an open-access article distributed under the terms of the Creative Commons Attribution License, which permits unrestricted use, distribution, and reproduction in any medium, provided the original author and source are credited. 
viral proteins or other macrophage products [19]. HIV-1 Tat-mediated brain lesions have also been reported to be associated with an increase of astrocytosis. Thus, astrogliosis is considered a striking pathological feature in HIV-1 infected brain [20]. Increasing evidence indicates astrocyte dysfunction during chronic HIV-1 CNS infection and immune activation play an important role in HIV-associated neuropathogenesis.

Astrocytes are identified by GFAP expression, which is an intermediate filament protein in the main processes and the soma. GFAP is generally considered to be a specific marker for astrogliotic response [21]. This protein is supposed to be involved in cell communication, cell migration, mitosis and cytoskeletal changes [22]. Astrocyte activation is characterized by elevated GFAP expression and this up-regulation is caused by diverse neurological insults [23], it is considered to be a hallmark feature observed in neurodegenerative diseases such as Alzheimer's disease, Parkinson's disease and HIV-1 infection [24]. Increased GFAP expression has also often been assumed to result from astrocyte proliferation [25]. Notably, astrocytes in GFAP knockout animals have interupted neuronal plasticity [26]. Astrocytosis is linked to productive infection of CNS and correlated with the cognitive disorders. It has also been shown that intracellular expression of HIV-1 Tat protein in astrocyte is linked to astrocytosis characterized by up-regulation expression of GFAP, astrocyte dysfunction, and neuronal death [24]. In addition, there is evidence that HIV-1 infected reactive astrocytes, together with activated microglia, may play a part in neuronal damage through the release of several inflammatory factors [18].

Activated astrocytes release neurotoxic factors including excitatory amino acids (EAAs) (such as glutamate) and TNF- $\alpha$ [27]. It is reported that gp120 up-regulates nuclear factor erythroid derived 2-related factor 2 (Nrf2), a basic leucine zipper transcription factor, which is involved in regulating the antioxidant defensive mechanism [28]. In addition, it has been shown that gp120 induces expression of IL-6 [29], IL-8 [30] and CCL5 in astrocytes [31] through a nuclear-kappa $\beta$-dependent mechanism. These results suggest that neuroinflammation in HIV1 -infected individuals could be mediated via the NF-k $\beta$ pathway. However, the dysfunction of activated astrocyte and their potential contributions to HIV-1 excitotoxicity and consequent CNS disorders is an area that warrants further insight.

\section{Gap Junction and HIV-1 Infected Astrocytes}

Gap junctions are the means of intercellular contact between two cells, which allows intercellular passage of ions and many molecules such as $\mathrm{K}^{+}$, second-messenger molecules, various metabolites and small peptides between the cytoplasm of adjacent cells. Astrocytes are interconnected to each other via gap junctions along with neurons and other glial cells via heterotypic gap junctions [32]. Gap junctions are composed of two aligned "hemi channels" or connexous, which form a specific pathway for the communication across the intercellular space and provide a specific route for propagating waves of ionic and metabolic signaling between cells [33]. On the other hand, despite the low numbers of HIV-infected astrocytes ( $8.2 \%$ in vivo and $4.7 \%$ in vitro using an SIV model) [34], significant changes in BBB integrity, $\mathrm{Ca}^{2+}$ concentration, glutamate clearance, or even cell death in un-infected astrocytes occur. It is proposed that HIV-infected astrocytes spread toxic signals to uninfected astrocytes by gap junction channels [35].

Gap junctions play a critical role in transmitting and thereby amplifying toxic signals originating from HIV-infected astrocytes to neighboring cells. It has been shown that Tat-induced BBB dysfunction is associated with altered expression of tight junction proteins zone occuldens (ZO-1), and junctional adhesion molecule-2 (JAM-2) [36]. It is also demonstrated that HIV infection of human astrocytes disrupts BBB integrity by a mechanism that is dependent on functional gap junctions [34,37]. Moreover, a low number of HIV-infected cells have been shown to affect profoundly BBB integrity, which is associated with dysregulating endothelial survival, stability of astrocytes end feet, and signaling [34]. Interestingly, Gap junction blockers abolished apoptosis in uninfected astrocytes [35].

It is noteworthy that gap junctions have critical contributions to astrocyte functionality. Astrocyte gap junctions have been shown to modulate ATP release and glutamate homeostasis [38,39]. In addition, gap junctions are also noted to create a buffering system that is responsible for the dissipation of focal increases of extracellular $\mathrm{K}^{+}$ resulting from neuronal activity [40]. Notably, BBB disruption induced by infected astrocytes was reduced by gap junction blockers, meaning that functional gap junction channels potentially mediate BBB integrity and permeability [34]. Therefore, mechanism of toxicity within the brain is generated by the low numbers of HIV-infected astrocytes and amplified to neighboring un-infected astrocytes by gap junctions, playing a significant role in HIV-1 CNS dysfunction [35].

\section{$\mathrm{K}^{+}$Channels in Astrocytes}

Astrocytes are multifunctional cells that are indispensable for neuronal survival and function, especially in the regulation of extracellular $\mathrm{K}^{+}$[41]. During neuronal activity, $\mathrm{K}^{+}$ions are transferred from the cytoplasm to the extracellular space and cause an increase in $\left[\mathrm{K}^{+}\right]_{0}$ which, if uncorrected, would induce the depolarization of neuronal membranes and the interruption of axonal conduction and synaptic transmission [42]. The clearance of extracellular $\mathrm{K}^{+}$by astrocytes takes place through a combination of active uptake, co-transport and through $\mathrm{K}^{+}$channels. Many studies have characterized that astrocytes have more hyperpolarized resting potential than that of neurons due to their expression of a wide variety of $\mathrm{K}^{+}$channels [43], which are located in astrocyte synapses and at end-food processes around capillaries [42] to mediate spatial $\mathrm{K}^{+}$buffering and regulate neural activities [44].

It has been strongly implicated that inward rectified $\mathrm{K}^{+}$channels (Kir) play roles to assist in spatial buffering of the extracellular $\mathrm{K}^{+}$ released during neuronal firing. Especially, Kir4.1 channels have been proven to be a specific glial Kir subunit [45], suggesting that they may have a specific function in glial $\mathrm{K}^{+}$regulation [46]. It is reported that Kir4.1 is responsible for hyperpolarization of astrocytes and is likely to play a critical role in $\mathrm{K}^{+}$buffering. Reduced astroglial Kir current was observed in many pathological conditions such as traumatic hippocampal glia [47], ischemic brain injury [48], cortical dysplasia [49] and in neurosurgical specimens from epilepsy patients [50]. In addition, Kir4.1 channels also appear to be responsible for the major resting $\mathrm{K}^{+}$conductance. Immunohistochemistry suggested that Kir4.1 is localized preferably on astroglial processes, ensheathing synapses and blood vessels [51]. Notably, genetic down-regulation of Kir4.1 impairs the ability of astrocytes to remove $\mathrm{K}^{+}$and glutamate in the extracellular space in vitro [52] and in vivo [53]. These results suggest a clear role for Kir4.1 in astrocyte $\mathrm{K}^{+}$buffering and glutamate uptake.

In contrast, the function of outward $\mathrm{K}^{+}$current in astrocyes is not very clear. It has been proposed that the outward $\mathrm{K}^{+}$current in astrocytes also contribute to special buffering, especially delayed rectified $\mathrm{K}^{+}$ current [54]. However, It remains to be determined on which type(s) of $\mathrm{K}^{+}$channels contribute(s) significantly to the capabilities of cortical 
astrocytes in $\mathrm{K}^{+}$buffering and glutamate uptake and how these $\mathrm{K}^{+}$ channel functions are affected during HIV-1 brain infectionleading to HAND pathogenesis.

\section{Glutamate Clearance}

Glutamate is the primarily excitatory neurotransmitter in the mammalian CNS [55]. Rapid removal of glutamate for the extracellular space is essential both for survival and normal functioning of neurons. Approximately $40 \%$ of all neuronal synapses in the brain are glutamatergic [56]. Thus, regulation of glutamate is crucial for optimal glutamatergic neurotransmission, which may otherwise cause to neuronal death by glutamate excitotoxicicty [55]. Uptake of glutamate is mainly mediated by transporters localized at astrocytic membranes. Although glutamate transporters are expressed in both astrocytes and neurons, astrocytes are the cells primarily responsible for glutamate uptake. It is estimated that astrocyte glutamate transporters are responsible for up to $90 \%$ of glutamate uptake in the brain [22].

Glutamate excitotoxicity has been proposed as one of potential mechanisms for neuronal injury in several neurological diseases including amyotropic lateral sclerosis, Alzheimer's disease [42] and HAND [57]. Exposure of cultured astrocytes to HIV-1 and gp120 impaired transport of extracellular glutamate by astrocytes [58]. Glutamate uptake inhibition was also observed during infection of feline astrocytes with feline immunodeficiency virus (FIV) [59] and in human astrocytes co-cultured with HTLV-I-infected T cells [60]. These results are in line with the findings of HIV-1 inhibition of glutamate transporter expression such as EAAT2 in astrocytes [58]. In addition, HIV-1 envelope proteins gp120 and gp41 have also been shown to impair glutamate uptake by primary human astrocytes [61], suggesting that disruption of astrocyte glutamate transport may be a common cytopathogenic activity mediated by some neurotropic retroviruses.

Glutamate transport is tightly regulated through transporter expression in astrocytes during brain development and in mature brain, and the levels of expression may be dys-regulated in diseases [62]. Five glutamate transporters of the SLC gene family, termed excitatory amino acid transporters 1 to 5 (EAAT1-5), have so far been identified in human brain [55]. Of these transporters, EAAT1 and 2, or glutamate transporter 1 (GLT-1) and L-glutamate/L-aspartate transporter (GLAST) in rodents, are expressed predominantly in astrocytes in the CNS and are believed to mediate most glutamate uptake in the brain. Expression of transporters EAAT1 and 2 was reduced in response to HIV-1, gp120 [58], and Tat [24]. These findings were in an agreement with the experimental results showing Tat-expression in astrocytes markedly impaired their ability in glutamate uptake [24]. These results indicate that HIV-1 and viral proteins may down-regulate expression and function of transporters EAAT1 and 2, resulting in glutamate excitotoxicity and neurodegeneration associated with HAND.

The synthesis and release of glutamine by astrocytes in part of a biochemical shuttle mechanism termed as the glutamate-glutamine cycle. After the release from the presynaptic terminal, glutamate is taken up mainly by astrocytes, and then converted to glutamine inside the astrocyte by the ATP-consuming reaction catalyzed by glutamine synthetase, an enzyme which is present in astrocytes but not in neurons [38]. It has been shown that GLT1 deficiency resulted in synaptic glutamate accumulation and subsequent excitotoxicity in mice [63]. Moreover, the reduction of EAAT1 and 2 expression and function in reactive astrocytes in response to HIV-1 and viral proteins can cause excess extracellular glutamate, which can induce increased intracellular calcium levels in astrocytes. This alteration, in turn, can elevate even more release of glutamate from cells in an autocrine manner [64].

There is now steady accumulating evidence that HIV-1 associated excitotoxicity is a direct result of brain glutamate dysregulation. This detrimental process is believed to involve activating N-methyl-Daspartic acid receptors (NMDARs) located in neuronal membrane by excess extracellular glutamate. The overactivation of NMDARs can cause an increase in intracellular $\mathrm{Ca}^{2+}[12]$ and the formation of free radical (nitro oxide and superoxide anion) [65]. The NMDAR over activation also causes activation of stress-associated protein kinases and caspases along with the production of proinflammatory lipids $[66,67]$ and release of additional neurotransmitter glutamate resulting in further injury [67]. All of these alterations may act synergistically to contribute to HIV-1 associated neuronal injury and ultimately HAND pathogenesis.

Since glutamate excitotoxicity is a factor in HIV neurotoxicity that culminates to HAND, potential ways to regulate the activation of the glutamate system for the treatment of HAND may be beneficial for the symptoms of HAND. Many strategies have been used over the years to facilitate the treatments by inhibition of glutaminase and glutaminate carboxypeptidase (the enzymes responsible for the production of glutamate), antagonists of glutamate receptors (NMDARs, AMPA, kainate and mGluR - responsible for glutamate excitotoxicity) or activation of glutamate transporters (e.g. EAAT1 and EAAT2 - responsible for mobilizing glutamate away from the synaptic cleft) $[64,68]$. In addition, there is preliminary experimental evidence that these approaches have therapeutic potential for ameliorating the HAND symptom repertoire [64]. Therefore, identification of specific inhibitors and further mechanistic insights are of significant important for the development of therapeutic strategies.

\section{Intracellular $\mathrm{Ca}^{2+}$ Concentration}

$\mathrm{Ca}^{2+}$ is a vital second messenger in astrocytes and involved in astrocyte response and communication. $\mathrm{Ca}^{2+}$ waves in astrocytes can transfer signals to neurons and modulate astrocyte activity [69]. Changes in the intracellular $\mathrm{Ca}^{2+}$ concentration play a critical role in many processes involved in the modulation of signal transduction, development and plasticity in the CNS. An increase of intracellular $\mathrm{Ca}^{2+}$ concentration is associated with cell excitability [70,71]. In addition, $\mathrm{Ca}^{2+}$ signals control many functions of astrocytes, especially exocytotic release of neurotransmitters glutamate or D-serine from astrocytes [72]. HIV-1 Tat can modulate intracellular $\mathrm{Ca}^{2+}$ concentration through activation of the endoplasmic reticulum (ER) pathway in astrocytic culture [73]. As a consequence, elevation of intracellular $\left[\mathrm{Ca}^{2+}\right]$ in astrocytes is able to initiate a similar response in nearby astrocytes in a regenerative wavelike fashion. Importantly, recent data shows that astrocyte $\left[\mathrm{Ca}^{2+}\right]$ modulates neuronal activity $[74,75]$, and $\mathrm{Ca}^{2+}$ elevation in astrocytes can lead to increases in neuronal $\left[\mathrm{Ca}^{2+}\right]_{\mathrm{i}}[76]$. Moreover, sustained elevation of the neuronal $\left[\mathrm{Ca}^{2+}\right]$ may trigger excessive NMDAR activation and consequent excitotoxicity [67].

The key pathway, that leads to $\left[\mathrm{Ca}^{2+}\right]_{\mathrm{I}}$ change, is the involvement of glutamate receptors. It has been demonstrated that the activation of glutamate receptors such as NMDA, AMPA, kainate receptors and metabotropic glutamate receptor subclass (mGluR1 and mGluR5) can change $\left[\mathrm{Ca}^{2+}\right]_{\mathrm{i}}[77]$. Released glutamate, in return, activates an inositol trisphosphate $\left(\mathrm{IP}_{3}\right)$ pathway, which can cause calcium release from intracellular storage. This increase can then be transferred and spread to neighboring astrocytes via gap junctions [42].

Since $\mathrm{Ca}^{2+}$ has many unique properties such as flexible co-ordination 
chemistry, high affinity for carboxylate oxygen and rapid binding kinetics, $\mathrm{Ca}^{2+}$ is considered as one of the most popular intracellular second messengers [33]. In particular, $\mathrm{Ca}^{2+}$ signals control several functions of astrocytes. It takes an active part in inducing exocytotic release of neurotransmitter from astrocytes as well as contributing in HIV neurotoxicity.

\section{Astrocytes and Blood Brain Barrier}

The blood brain barrier (BBB) is a specialized system of brain microvascular endothelial cells (BMECs) that function to separate circulating blood from brain extracellular fluid, which prevents toxic substances present in the blood from entering the brain and supplies brain tissues with nutrients [22]. The BMECs are surrounded by astrocytes and their end feet processes, which contribute importantly to the development and function of the BBB [34]. Furthermore, astrocytes are supposed to be involved in maintaining the microenvironment that preserves the functionality of BMECs [78]. It has been accepted that HIV-1 neuroinvasion occurs as a result of HIV-1-infected monocytes across the $\mathrm{BBB}$, and the dysregulation of the $\mathrm{BBB}$ permeability during and after neuroinvasion has a critical impact on HIV-1 neuropathogenesis [22]. Therefore, the role of astrocytes in the BBB is one of the many motives to understand the molecular mechanisms associated with HIV-1 neuroinvastion and neuropathological process.

Astrocytic end feet, which contact the BBB, are rich in glutamate transporters and potassium channels. These proteins are involved

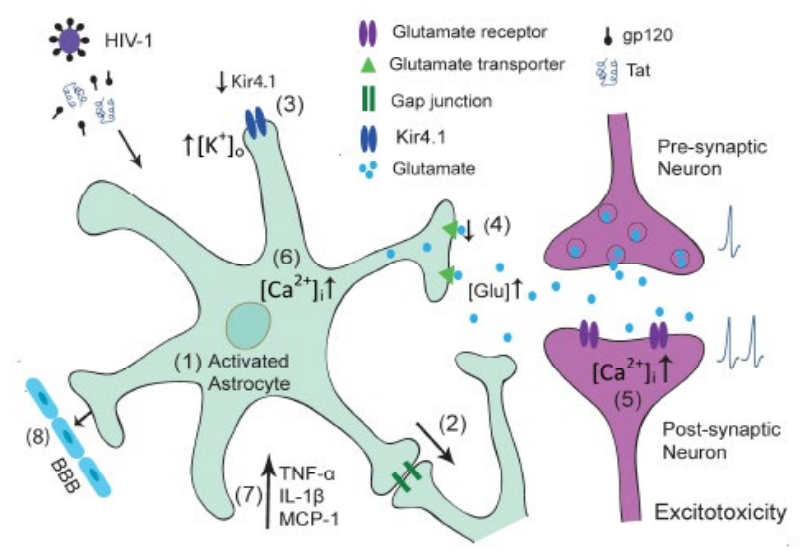

Figure 1: Schematic representation of astrocytic dysfunctions in HIV-1 neurotoxicity. (1) Astrocytes respond to HIV infection and viral proteins by rapid activation. (2) This response spreads and amplifies to surrounding astrocytes via gap junctions, which take part in modulating ATP release and glutamate and potassium homeostasis. (3) Reduced Kir4.1 expression and function impairs the ability of activated astrocytes to remove $\mathrm{K}^{+}$in extracellular space and cause an increase in $\left[\mathrm{K}^{+}\right]_{0}$, which would induce the depolarization of neuronal membranes and the interruption of axonal conduction and synaptic transmission. (4) Down regulation of glutamate expression and function results in glutamate excitotoxicity, which eventually may lead to neuronal injury and death. (5) This detrimental process is induced by overactivation of glutamate receptors located in neuronal membranes and causes an increase in intracellular $\mathrm{Ca}^{2+}$ levels, resulting in further injury. (6) Elevation of intracellular $\mathrm{Ca}^{2+}$ in astrocytes can also lead to increase in neuronal $\mathrm{Ca}^{2+}$, which may trigger excessive glutamate receptor activation and consequent excitotoxicity. (7) In addition, HIV-1 induced activated astrocytes secrete proimflammatory cytokines and chemokines, which may cause neuronal dysfunction or injury. (8) Reduced glutamate uptake, impaired potassium channel activity and elevated intracellular $\mathrm{Ca}^{2+}$ affect the BBB integrity and permeability resulting in increased penetration of HIV into the CNS. These alterations are likely acting synergistically to contribute to the accumulation of neurotoxicity and potentially play a role in HIV-1 induced neuropathogenesis. in ion and glutamate homeostasis [79]. As indicated earlier, HIV1 proteins alter glutamate uptake and potassium channel activity in astrocytes, thus, it is conceivable that this alteration may affect the BBB's integrity. In another aspect, HIV-1 Tat and gp120 has been shown to elevate intracellular $\mathrm{Ca}^{2+}$. This increase in astrocytic $\mathrm{Ca}^{2+}$ may induce the release of COX-1 metabolic products, resulting in vasodilatation of capillaries [80]. It is reported that astrocytes secret many molecular mediators such as NO, prostaglandin (PGE), and arachidonic acid (AA) that can increase or decrease CNS blood vessel diameter and blood flow. Astrocytes also produce and release several angiogenic factors such as angiopoetin 1 and neurotrophins that potentially play a part in the development of new brain capillary functions. Notably, it has been observed that HIV-1 Tat and gp120 alter the expression of tight junction proteins (TJ) which is associated with astrocytes along the barrier, resulting in increasing permeability of the BBB [22,81]. This evidence supports the idea that astrocytes would likely contribute to the effect of HIV-1 and its proteins on the BBB integrity, resulting in increased penetration of HIV into the CNS. The possible mechanism of this contribution to HIV-1 disease progression is an important point for future investigation.

\section{Astrocyte-neuron Interaction}

Communication between astrocytes and neurons is bidirectional. It is now accepted that astrocytes and neurons establish a highly dynamic reciprocal relationship which influences CNS growth, morphology, repair and aging. Therefore, it is highly conceivable that dysfunction of astrocytes could potentially contribute to neurological diseases. Recent studies have suggested that reactive astrocytes gain neurotoxic properties. Notably, excitotoxicity in the brain is a consequence of dysregulated mechanisms in astrocytes, which affect neurons when neither the astrocytes nor the neurons are direct targets of viral infection.

Consistent with such a variety of crucial functions exerted by astrocytes to support neurons, astrocyte impairment has been proven to contribute to neuronal dysfunction in various neurodegeneration diseases, such as amyotrophic lateral sclerosis, Huntington disease and Alzheimer's disease [3]. In vitro studies have shown that Tat released from astrocytes produces mitochondrial dysfunction and cell death in neurons [82]. Infection of astrocytes by HIV-1 also leads to hijacking of protein synthesis and consequent reduction of trophic factors production [3]. In support of these findings, it has been shown that supernatant from Tat-expressing astrocytes were neurotoxic to neurons [24].

In addition to having direct impacts on synaptic transmission through release of transmitters, called gliotransmitters, astrocytes have the potential to exert long-term and powerful influences on synaptic function via release of growth factors and other bioactive molecules such as cytokines. It has been reported that HIV-1 Tat and HIV-1 gp120 activate astrocytes to secrete proimflammatory cytokines TNF- $\alpha$ [12], IL-1, IL-1 $\beta$ [83], proimflamatory chemokines MCP-1 and IP-10,as well as neurotoxic nitro oxide (NO) [2]. Cytokines, such as TNF- $\alpha$, have been found to influence homeostatic synaptic scaling by inducing the addition of AMPA receptors, an ionotropic transmembrane receptor for glutamate, at post-synaptic membranes. Moreover, reactive astrocytes hinder regeneration of damaged neuronal circuits by secreting neurondevelopmental inhibitor and glycosaminoglycans, which physically block growth cone extension [84]. All of which can potentially contribute to the inflammatory brain injury [85]. 


\section{Summary and Prospects}

In the brain, astrocytes represent a main population of the nonneuronal cell type and fulfill a crucial role in both physiological and pathological conditions. In a physiological environment, astrocytes regulate brain homeostasis, synaptic transmission and plasticity to protect neurons against toxic compounds, and support metabolically to ensure their proper functioning. Under pathological condition such as HAND, astrocytes are involved in HIV-1 neuropathogenesis. A series of changes in astrocyte functions are detected during HIV-1 infection including, but not limited to, astrocytosis, reduced glutamate uptake and altered ion homeostasis (Figure 1), which may be amplified via gap junctions to surrounding astrocytes. As astrocytes are in close contact with neurons and are capable of sensing neuronal activity, the impairment on aforementioned astrocyte functions can cause neuronal dysfunction or injury by increased extracellular $\mathrm{K}^{+}$and glutamate concentration or intracellular $\mathrm{Ca}^{2+}$ concentration of neurons, or by release of proinflammatory chemokines (Figure 1), eventually development of HAND. Therefore, modulation of astrocyte functions may have therapeutic potential for HIV-1-associated neurological disorders as well as other neurodegenerative disorders in which astrocyte dysfunction plays a role in the pathogenesis.

\section{Acknowledgement}

The authors thank Ms. Cassie Huang, Mr. Cory Collins and Thieu Phan for reading the manuscript and helpful comments. This work was supported by NIH grants R01 NS063878 and R01 NS077873 to HX.

\section{References}

1. Davis LE, Hjelle BL, Miller VE, Palmer DL, Llewellyn AL, et al. (1992) Early vira brain invasion in iatrogenic human immunodeficiency virus infection. Neurology 42: $1736-1739$

2. McArthur JC, Brew BJ, Nath A (2005) Neurological complications of HIV infection. Lancet Neurol 4: 543-555.

3. De Keyser J, Mostert JP, Koch MW (2008) Dysfunctional astrocytes as key players in the pathogenesis of central nervous system disorders. J Neurol Sci 267: 3-16.

4. Antinori A, Arendt G, Becker JT, Brew BJ, Byrd DA, et al. (2007) Updated research nosology for HIV-associated neurocognitive disorders. Neurology 69 : 1789-1799.

5. Jaeger LB, Nath A (2012) Modeling HIV-associated neurocognitive disorders in mice: new approaches in the changing face of HIV neuropathogenesis. Dis Model Mech 5: 313-322.

6. del Palacio M, Alvarez S, Muñoz-Fernández MÁ (2012) HIV-1 infection and neurocognitive impairment in the current era. Rev Med Virol 22: 33-45.

7. Ances BM, Ellis RJ (2007) Dementia and neurocognitive disorders due to HIV-1 infection. Semin Neurol 27: 86-92.

8. Glass JD, Fedor H, Wesselingh SL, McArthur JC (1995) Immunocytochemical quantitation of human immunodeficiency virus in the brain: correlations with dementia. Ann Neurol 38: 755-762.

9. Takahashi K, Wesselingh SL, Griffin DE, McArthur JC, Johnson RT, et al. (1996) Localization of HIV-1 in human brain using polymerase chain reaction/in situ hybridization and immunocytochemistry. Ann Neurol 39: 705-711.

10. Saito Y, Sharer LR, Epstein LG, Michaels J, Mintz M, et al. (1994) Overexpression of nef as a marker for restricted HIV-1 infection of astrocytes in postmortem pediatric central nervous tissues. Neurology 44: 474-481.

11. Brack-Werner R (1999) Astrocytes: HIV cellular reservoirs and important participants in neuropathogenesis. AIDS 13: 1-22.

12. Kaul M, Garden GA, Lipton SA (2001) Pathways to neuronal injury and apoptosis in HIV-associated dementia. Nature 410: 988-994.

13. Jing T, Wu L, Borgmann K, Surendran S, Ghorpade A, et al. (2010) Soluble factors from IL-1 $\hat{1}^{2}$-stimulated astrocytes activate NR1a/NR2B receptors: implications for HIV-1-induced neurodegeneration. Biochem Biophys Res Commun 402: 241-246
14. Boven LA, Vergnolle N, Henry SD, Silva C, Imai Y, et al. (2003) Up-regulation of proteinase-activated receptor 1 expression in astrocytes during HIV encephalitis. J Immunol 170: 2638-2646.

15. Dou H, Morehead J, Bradley J, Gorantla S, Ellison B, et al. (2006) Neuropathologic and neuroinflammatory activities of HIV-1-infected human astrocytes in murine brain. Glia 54: 81-93.

16. Sofroniew MV, Vinters HV (2010) Astrocytes: biology and pathology. Acta Neuropathol 119: 7-35.

17. Gorry PR, Ong C, Thorpe J, Bannwarth S, Thompson KA, et al. (2003) Astrocyte infection by HIV-1: mechanisms of restricted virus replication, and role in the pathogenesis of HIV-1-associated dementia. Curr HIV Res 1: 463-473.

18. Deshpande M, Zheng J, Borgmann K, Persidsky R, Wu L, et al. (2005) Role of activated astrocytes in neuronal damage: potential links to HIV-1-associated dementia. Neurotox Res 7: 183-192.

19. Verma AS, Singh UP, Dwivedi PD, Singh A (2010) Contribution of CNS cells in NeuroAIDS. J Pharm Bioallied Sci 2: 300-306.

20. Mamik MK, Banerjee S, Walseth TF, Hirte R, Tang L, et al. (2011) HIV-1 and $\mathrm{IL}-1 \hat{\mid}^{2}$ regulate astrocytic CD38 through mitogen-activated protein kinases and

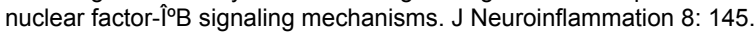

21. Verkhratsky A, Rodríguez JJ, Parpura V (2013) Astroglia in neurologica diseases. Future Neurol 8: 149-158.

22. Cisneros IE, Ghorpade A (2012) HIV-1, methamphetamine and astrocyte glutamate regulation: combined excitotoxic implications for neuro-AIDS. Curr HIV Res 10: 392-406.

23. Vanzani MC, lacono RF, Caccuri RL, Troncoso AR, Berria MI (2006) Regional differences in astrocyte activation in HIV-associated dementia. Medicina (B Aires) 66: 108-112.

24. Zhou BY, Liu Y, Kim Bo, Xiao Y, He JJ (2004) Astrocyte activation and dysfunction and neuron death by HIV-1 Tat expression in astrocytes. Mol Cell Neurosci 27: 296-305

25. Li J, Ding YH, Rafols JA, Lai Q, McAllister JP 2nd, et al. (2005) Increased astrocyte proliferation in rats after running exercise. Neurosci Lett 386: 160164

26. McCall MA, Gregg RG, Behringer RR, Brenner M, Delaney CL, et al. (1996) Targeted deletion in astrocyte intermediate filament (Gfap) alters neuronal physiology. Proc Natl Acad Sci U S A 93: 6361-6366.

27. Bezzi P, Domercq M, Brambilla L, Galli R, Schols D, et al. (2001) CXCR4activated astrocyte glutamate release via TNFalpha: amplification by microglia triggers neurotoxicity. Nat Neurosci 4: 702-710.

28. Reddy PV, Gandhi N, Samikkannu T, Saiyed Z, Agudelo M, et al. (2012) HIV-1 gp120 induces antioxidant response element-mediated expression in primary astrocytes: role in HIV associated neurocognitive disorder. Neurochem Int 61 : 807-814.

29. Shah A, Verma AS, Patel KH, Noel R, Rivera-Amill V, et al. (2011) HIV-1 gp120 induces expression of IL-6 through a nuclear factor-kappa B-dependent mechanism: suppression by gp120 specific small interfering RNA. PLoS One 6: e21261.

30. Shah A, Kumar A (2010) HIV-1 gp120-mediated increases in IL-8 production in astrocytes are mediated through the NF-10 ${ }^{\circ} \mathrm{B}$ pathway and can be silenced by gp120-specific siRNA. J Neuroinflammation 7: 96.

31. Shah A, Singh DP, Buch S, Kumar A (2011) HIV-1 envelope protein gp120 up regulates CCL5 production in astrocytes which can be circumvented by inhibitors of NF-Î̊B pathway. Biochem Biophys Res Commun 414: 112-117.

32. Williams A, Piaton G, Lubetzki C (2007) Astrocytes--friends or foes in multiple sclerosis? Glia 55: 1300-1312.

33. Parpura V, Verkhratsky A (2012) Homeostatic function of astrocytes: $\mathrm{Ca}(2+)$ and $\mathrm{Na}(+)$ signalling. TransI Neurosci 3: 334-344.

34. Eugenin EA, Clements JE, Zink MC, Berman JW (2011) Human immunodeficiency virus infection of human astrocytes disrupts blood-brain barrier integrity by a gap junction-dependent mechanism. J Neurosci 31: 94569465.

35. Eugenin EA, Berman JW (2007) Gap junctions mediate human immunodeficiency virus-bystander killing in astrocytes. J Neurosci 27: 12844 12850. 
36. Toborek M, Lee YW, Flora G, Pu H, András IE, et al. (2005) Mechanisms of the blood-brain barrier disruption in HIV-1 infection. Cell Mol Neurobiol 25: 181199.

37. Mishra R, Singh SK (2013) HIV-1 Tat C modulates expression of miRNA-101 to suppress VE-cadherin in human brain microvascular endothelial cells. $J$ Neurosci 33: 5992-6000

38. Ye ZC, Wyeth MS, Baltan-Tekkok S, Ransom BR (2003) Functional hemichannels in astrocytes: a novel mechanism of glutamate release. $\mathrm{J}$ Neurosci 23: 3588-3596.

39. Stout CE, Costantin JL, Naus CC, Charles AC (2002) Intercellular calcium signaling in astrocytes via ATP release through connexin hemichannels. J Biol Chem 277: 10482-10488.

40. Dermietzel R, Hertberg EL, Kessler JA, Spray DC (1991) Gap junctions between cultured astrocytes: immunocytochemical, molecular, and electrophysiological analysis. J Neurosci 11: 1421-1432.

41. Walz W (2000) Role of astrocytes in the clearance of excess extracellular potassium. Neurochem Int 36: 291-300.

42. Maragakis NJ, Rothstein JD (2006) Mechanisms of Disease: astrocytes in neurodegenerative disease. Nat Clin Pract Neurol 2: 679-689.

43. Olsen ML, Higashimori H, Campbell SL, Hablitz JJ, Sontheimer H (2006) Functional expression of Kir4.1 channels in spinal cord astrocytes. Glia 53: 516-528.

44. Nagao Y, Harada Y, Mukai T, Shimizu S, Okuda A, et al. (2013) Expressional analysis of the astrocytic Kir4.1 channel in a pilocarpine-induced temporal lobe epilepsy model. Front Cell Neurosci 7: 104.

45. Takumi T, Ishii T, Horio Y, Morishige K, Takahashi N, et al. (1995) A novel ATPdependent inward rectifier potassium channel expressed predominantly in glial cells. J Biol Chem 270: 16339-16346.

46. Kalsi AS, Greenwood K, Wilkin G, Butt AM (2004) Kir4.1 expression by astrocytes and oligodendrocytes in CNS white matter: a developmental study in the rat optic nerve. J Anat 204: 475-485.

47. D'Ambrosio R, Maris DO, Grady MS, Winn HR, Janigro D (1999) Impaired $\mathrm{K}(+)$ homeostasis and altered electrophysiological properties of post-traumatic hippocampal glia. J Neurosci 19: 8152-8162.

48. Köller H, Schroeter M, Jander S, Stoll G, Siebler M (2000) Time course of inwardly rectifying $\mathrm{K}(+)$ current reduction in glial cells surrounding ischemic brain lesions. Brain Res 872: 194-198.

49. Bordey A, Lyons SA, Hablitz JJ, Sontheimer H (2001) Electrophysiological characteristics of reactive astrocytes in experimental cortical dysplasia. J Neurophysiol 85: 1719-1731.

50. Hinterkeuser S, Schröder W, Hager G, Seifert G, Blümcke I, et al. (2000) Astrocytes in the hippocampus of patients with temporal lobe epilepsy display changes in potassium conductances. Eur J Neurosci 12: 2087-2096.

51. Higashi K, Fujita A, Inanobe A, Tanemoto M, Doi K, et al. (2001) An inwardly rectifying $\mathrm{K}(+)$ channel, Kir4.1, expressed in astrocytes surrounds synapses and blood vessels in brain. Am J Physiol Cell Physiol 281: C922-931.

52. Kucheryavykh YV, Kucheryavykh LY, Nichols CG, Maldonado HM, Baksi K, et al. (2007) Downregulation of Kir4.1 inward rectifying potassium channel subunits by RNAi impairs potassium transfer and glutamate uptake by cultured cortical astrocytes. Glia 55: 274-281.

53. Djukic B, Casper KB, Philpot BD, Chin LS, McCarthy KD (2007) Conditional knock-out of Kir4.1 leads to glial membrane depolarization, inhibition of potassium and glutamate uptake, and enhanced short-term synaptic potentiation. J Neurosci 27: 11354-11365.

54. Sontheimer H (1994) Voltage-dependent ion channels in glial cells. Glia 11: 156-172.

55. Danbolt NC (2001) Glutamate uptake. Prog Neurobiol 65: 1-105.

56. Richter JD, Klann E (2009) Making synaptic plasticity and memory last: mechanisms of translational regulation. Genes Dev 23: 1-11.

57. Sabri F, Titanji K, De Milito A, Chiodi F (2003) Astrocyte activation and apoptosis: their roles in the neuropathology of HIV infection. Brain Pathol 13: 84-94.

58. Wang Z, Pekarskaya O, Bencheikh M, Chao W, Gelbard HA, et al. (2003) Reduced expression of glutamate transporter EAAT2 and impaired glutamate transport in human primary astrocytes exposed to HIV-1 or gp120. Virology 312: $60-73$.
59. Billaud JN, Selway D, Yu N, Phillips TR (2000) Replication rate of feline immunodeficiency virus in astrocytes is envelope dependent: implications for glutamate uptake. Virology 266: 180-188.

60. Henriksen SJ, Prospero-Garcia O, Phillips TR, Fox HS, Bloom FE, et al. (1995) Feline immunodeficiency virus as a model for study of lentivirus infection of the central nervous system. Curr Top Microbiol Immunol 202: 167-186.

61. Patton HK, Zhou ZH, Bubien JK, Benveniste EN, Benos DJ (2000) gp120induced alterations of human astrocyte function: $\mathrm{Na}(+) / \mathrm{H}(+)$ exchange, $\mathrm{K}(+)$ conductance, and glutamate flux. Am J Physiol Cell Physiol 279: C700-708.

62. Maragakis NJ, Rothstein JD (2001) Glutamate transporters in neurologic disease. Arch Neurol 58: 365-370.

63. Tanaka K, Watase K, Manabe T, Yamada K, Watanabe M, et al. (1997) Epilepsy and exacerbation of brain injury in mice lacking the glutamate transporter GLT1. Science 276: 1699-1702.

64. Potter MC, Figuera-Losada M, Rojas C, Slusher BS (2013) Targeting the glutamatergic system for the treatment of HIV-associated neurocognitive disorders. J Neuroimmune Pharmacol 8: 594-607.

65. Lipton SA, Sucher NJ, Kaiser PK, Dreyer EB (1991) Synergistic effects of HIV coat protein and NMDA receptor-mediated neurotoxicity. Neuron 7: 111-118.

66. Nicotera P, Ankarcrona M, Bonfoco E, Orrenius S, Lipton SA (1997) Neuronal necrosis and apoptosis: two distinct events induced by exposure to glutamate or oxidative stress. Adv Neurol 72: 95-101.

67. Kaul M, Lipton SA (2006) Mechanisms of neuronal injury and death in HIV-1 associated dementia. Curr HIV Res 4: 307-318.

68. Toggas SM, Masliah E, Mucke L (1996) Prevention of HIV-1 gp120-induced neuronal damage in the central nervous system of transgenic mice by the NMDA receptor antagonist memantine. Brain Res 706: 303-307.

69. Ricci G, Volpi L, Pasquali L, Petrozzi L, Siciliano G (2009) Astrocyte-neuron interactions in neurological disorders. J Biol Phys 35: 317-336.

70. Cornell-Bell AH, Finkbeiner SM, Cooper MS, Smith SJ (1990) Glutamate induces calcium waves in cultured astrocytes: long-range glial signaling. Science 247: 470-473

71. Charles AC, Merrill JE, Dirksen ER, Sanderson MJ (1991) Intercellular signaling in glial cells: calcium waves and oscillations in response to mechanical stimulation and glutamate. Neuron 6: 983-992.

72. Jellinger KA (2009) Astrocytes in (Patho) Physiology of the Nervous System. European Journal of Neurology 16: e164.

73. Köller H, Schaal H, Freund M, Garrido SR, von Giesen HJ, et al. (2001) HIV 1 protein Tat reduces the glutamate-induced intracellular $\mathrm{Ca} 2+$ increase in cultured cortical astrocytes. Eur J Neurosci 14: 1793-1799.

74. Agulhon C, Fiacco TA, McCarthy KD (2010) Hippocampal short- and long-term plasticity are not modulated by astrocyte $\mathrm{Ca}+$ signaling. Science $327: 1250$ 1254.

75. Nedergaard M, Verkhratsky A (2012) Artifact versus reality--how astrocytes contribute to synaptic events. Glia 60: 1013-1023.

76. Nedergaard M (1994) Direct signaling from astrocytes to neurons in cultures of mammalian brain cells. Science 263: 1768-1771.

77. Chen J, Backus KH, Deitmer JW (1997) Intracellular calcium transients and potassium current oscillations evoked by glutamate in cultured rat astrocytes. J Neurosci 17: 7278-7287.

78. Strazza M, Pirrone V, Wigdahl B, Nonnemacher MR (2011) Breaking down the barrier: the effects of HIV-1 on the blood-brain barrier. Brain Res 1399: 96-115.

79. Blackburn D, Sargsyan S, Monk PN, Shaw PJ (2009) Astrocyte function and role in motor neuron disease: a future therapeutic target? Glia 57: 1251-1264.

80. Takano T, Tian GF, Peng W, Lou N, Libionka W, et al. (2006) Astrocyte-mediated control of cerebral blood flow. Nat Neurosci 9: 260-267.

81. Mahajan SD, Aalinkeel R, Sykes DE, Reynolds JL, Bindukumar B, et al. (2008) Methamphetamine alters blood brain barrier permeability via the modulation of tight junction expression: Implication for HIV-1 neuropathogenesis in the context of drug abuse. Brain Res 1203: 133-148.

82. Chauhan A, Turchan J, Pocernich C, Bruce-Keller A, Roth S, et al. (2003) Intracellular human immunodeficiency virus Tat expression in astrocytes promotes astrocyte survival but induces potent neurotoxicity at distant sites via axonal transport. J Biol Chem 278: 13512-13519. 
83. Nottet HS, Jett M, Flanagan CR, Zhai QH, Persidsky Y, et al. (1995) A regulatory role for astrocytes in HIV-1 encephalitis. An overexpression of eicosanoids, platelet-activating factor, and tumor necrosis factor-alpha by activated HIV-1infected monocytes is attenuated by primary human astrocytes. J Immunol 154 3567-3581.

84. Kang WH, Simon MJ, Gao S, Banta S, Morrison B 3rd (2011) Attenuation of astrocyte activation by TAT-mediated delivery of a peptide JNK inhibitor. J Neurotrauma 28: 1219-1228.
85. Wang T, Gong N, Liu J, Kadiu I, Kraft-Terry SD, et al. (2008) Proteomic modeling for HIV-1 infected microglia-astrocyte crosstalk. PLoS One 3: e2507.

Citation: Ton $\mathrm{H}$, Xiong $\mathrm{H}$ (2013) Astrocyte Dysfunctions and HIV-1

Neurotoxicity. J AIDS Clin Res 4: 255. doi: 10.4172/2155-6113.1000255 